\title{
Silencing of the TPM1 gene induces radioresistance of glioma $\mathrm{U} 251$ cells
}

\author{
HUA-QING DU $^{1,2^{*}}$, YING WANG $^{1 *}$, YAO JIANG $^{1}$, CHEN-HAN WANG $^{1}$, \\ TAO ZHOU ${ }^{1}$, HONG-YI LIU ${ }^{1}$ and HONG XIAO ${ }^{1}$ \\ ${ }^{1}$ Department of Neuro-Psychiatric Institute, Nanjing Brain Hospital Affiliated to Nanjing Medical University, \\ Nanjing, Jiangsu 210029; ${ }^{2}$ Department of Pharmacy, Nanjing Maternal and Child Care Hospital \\ Affiliated to Nanjing Medical University, Nanjing, Jiangsu 210004, P.R. China
}

Received January 17, 2015; Accepted February 27, 2015

DOI: $10.3892 /$ or.2015.3906

\begin{abstract}
The present study was designed to investigate the relationship between tropomyosin 1 (TPM1) and radioresistance in human U251 cells. Radioresistant U251 (RR-U251) cells were established by repeated small irradiating injury. TPM1 levels in the U251 and RR-U251 cells were inhibited by transfection with TPM1-short hairpin RNA (shRNA) while overexpression was induced by treatment with pcDNA3.1-TPM1. The radiosensitivity of the $\mathrm{U} 251$ and $\mathrm{R} R-\mathrm{U} 251$ cells and the plasmid-transfected cells was evaluated by cell viability, migration and invasion assays. Cell apoptosis was also examined in vitro. The radiosensitivity of U251 xenografts was observed by tumor growth curve after radiotherapy in an in vivo experiment. Western blotting and immunohistochemistry were used to detect the level of TPM1 in vivo. The expression of TPM1 was significantly decreased in the RR-U251 cells, which may be correlated with the radioresistance of the glioma U251 cells. In the TPM1-silenced RR-U251 and TPM1-silenced U251 cells, cell viability, migration and invasion ability were significantly increased, and the rate of cell apoptosis was decreased. Consistent with these results, in the TPM1-overexpressing U251 and RR-U251 cells, cell viability, migration and invasion abilities were markedly decreased, and increased apoptosis was noted when compared to the control group. Tumor growth of the U251 xenografts was significantly inhibited following treatment with pcDNA3.1-TPM1 combined with radiotherapy. Taken together, these results indicate that TPM1 may be one mechanism underlying radiation resistance, and TPM1 may be a potential target for overcoming the radiation resistance in glioma.
\end{abstract}

Correspondence to: Professor Hong Xiao or Professor Hong-Yi Liu, Department of Neuro-Psychiatric Institute, Nanjing Brain Hospital Affiliated to Nanjing Medical University, 264 Guangzhou Road, Nanjing, Jiangsu 210029, P.R. China

E-mail: xhnkyy123@163.com

E-mail: hyliu18@126.com

*Contributed equally

Key words: glioblastoma, U251, radioresistance, TPM1

\section{Introduction}

Malignant gliomas, the most common primary brain tumors, are highly invasive and neurologically destructive neoplasms with an extremely poor prognosis due to limitations in the complete surgical resection of the mass and the limited activity of present therapeutic agents (1-3). The median length of survival of patients receiving no further therapy is usually $<1$ year from the time of diagnosis (4). Yet, surgical treatment alone does not affect the median length of survival, while radiotherapy has been shown to prolong the median survival for 6-8 months (5). Hence, radiation therapy continues to play a central role in the management of brain tumors $(6,7)$. Unfortunately, most patients receiving radiation therapy still develop tumor recurrence in the proximity of the primary site. Recent studies have reported that glioblastoma (GBM) recurrence hampers effective treatment.

Determining the cellular, molecular and biochemical mechanisms underlying radiation resistance is critical for the successful treatment of glioma tumors $(8,9)$.

Tropomyosins (TMs) are found as a family of actin filament binding proteins in skeletal muscle. The TM family is divided into two major groups: high-molecular-weight (HMW) and low-molecular-weight (LMW) TMs. Tropomyosin 1 (TPM1) is a gene that encodes isoforms of the HMW TMs (10). HMW TM isoforms are downregulated in various types of tumors, such as breast, urinary bladder and neuroblastoma tumors (11-14). HMW TMs can regulate the proliferation, motility, invasion and metastasis of tumor cells (15). TPM1 is the most important TM in breast cancer, where it functions as a suppressor of cell transformation (16). However, the status and significance of TPM1 expression in glioma have never been investigated.

In the present study, we were able to go beyond a simple correlation between TPM1 and radioresistance. These results should encourage future research on gene therapy as potential anticancer agents in glioma.

\section{Materials and methods}

Establishment of radioresistant U251 (RR-U251) cells. U251 cells were irradiated as a monolayer culture by $\gamma$-rays of a ${ }^{60} \mathrm{Co}$ 
source at $0.5 \mathrm{~Gy} / \mathrm{min}$ for $10 \mathrm{~min}$ each time until the accumulation was $60 \mathrm{~Gy}$.

Cell culture. The human glioma U251 cells were obtained from KeyGene (Nanjing, China). All cells were grown in Dulbecco's modified Eagle's medium (DMEM) containing $10 \%$ fetal bovine serum, penicillin $(100 \mathrm{U} / \mathrm{ml})$, and streptomycin $(100 \mu \mathrm{g} / \mathrm{ml})$ in a $5 \% \mathrm{CO}_{2}$ incubator at $37^{\circ} \mathrm{C}$.

PreparationofshorthairpinRNA(shRNA)andpcDNA3.1-TPM1. According to the target sequences of the TPM1 gene (gene ID: 7168), interferential sequence TPM1-shRNA was designed. The TPM1 shRNA sequences were: 5'-CAC CGG AAG ACA GAT ATG AGG AAG ATT CAA GAG ATC TTC CTC ATA TCT GTC TTC CTT TTT TG-3' (forward) and 5'-GAT CCA AAA AAG GAA GAC AGA TAT GAG GAA GAT CTC TTG AAT CTT CCT CAT ATC TGT CTT CC-3' (reverse). Oligonucleotides were synthesized by GenePharma Company (Shanghai, China), and scrambled shRNA sequences served as the negative control. pcDNA3.1-TPM1 was also obtained from the GenePharma Company.

Transfection. Transient transfections were performed using Lipofectamine 2000 according to the manufacturer's instructions (Invitrogen). Briefly, U251 or RR-U251 cells $\left(5 \times 10^{6}\right)$ were plated in a 6 -well plate supplied with $2 \mathrm{ml}$ medium for $24 \mathrm{~h}$. The cells were then washed twice with serum- and antibiotic-free DMEM. Plasmids/negative control plasmid and Lipofectamine 2000 were premixed for $25 \mathrm{~min}$ and applied to the cells in $2 \mathrm{ml}$ of serum- and antibiotic-free DMEM. After $6 \mathrm{~h}$, the serum- and antibiotic-free DMEM was replaced with $2 \mathrm{ml}$ of complete medium. The positively transfected cells were selected in the presence of the neomycin-analogue G418 (400 mg/ml).

Irradiation. The normal U251 and RR-U251 cells, transfected with shRNA-TPM1 or pcDNA3.1-TPM1 or the untreated cells were irradiated with a range of single doses of 5 Gy of $\gamma$-rays using a ${ }^{60} \mathrm{Co}$ self-shielded irradiator at a dose rate of $0.5 \mathrm{~Gy} /$ min. All irradiations were performed at room temperature. After irradiation, the culture plates were returned to the incubator and maintained at $37^{\circ} \mathrm{C}$.

Western blot analysis. Cells were lysed, and equal amounts of protein were electrophoresed on a $12 \%$ SDS-polyacrylamide gel and subsequently transferred to polyvinylidene fluoride (PVDF) membranes. The membranes were blocked in 5\% skim milk in phosphate-buffered saline (PBS) containing $0.1 \%$ Tween-20 (PBST) for $1 \mathrm{~h}$ at room temperature. The membranes were incubated with the following primary antibodies at $4^{\circ} \mathrm{C}$ overnight: TPM1 (1:400, ab55915; Abcam, Hong Kong, SAR, China) and $\alpha$-tubulin (1:500; Beijing Biosynthesis Biotechnology, China). The membranes were then washed 3 times with PBST, and incubated with horseradish peroxidase (HRP)-conjugated secondary antibody (1:2,000; Beijing Biosynthesis Biotechnology) for $45 \mathrm{~min}$ at room temperature. After three PBST washes, the membranes were developed using ECL Plus (Millipore, MA, USA) and exposed to X-ray film for visualization of the protein bands. $\alpha$-tubulin was used as an internal loading control.
Cell proliferation assay. Cell viability was assayed by the MTT assay. After radiotherapy, the cells were seeded on a 96-well plate at $1 \times 10^{4}$ cells/well and incubated for $48 \mathrm{~h}$. Then, $5 \mathrm{mg} / \mathrm{ml}$ MTT was added into each well for $4 \mathrm{~h}$. The supernatants were carefully aspirated, and the formazan crystals were dissolved using dimethyl sulfoxide (DMSO). Absorbance was measured at $570 \mathrm{~nm}$ with a microplate reader. The results were representative of the relative survival rate. The relative cell survival rate was measured by the formula:

Cell viability $(100 \%)=\mathrm{OD}_{\text {treatment }} / \mathrm{OD}_{\text {control }} \times 100 \%$ (formula 1 ).

Wound-healing experiments. The wound-healing assay was used to evaluate cell migration ability. After radiotherapy, U251 and RR-U251 cells treated with TPM1-shRNA or pcDNA3.1-TPM1 were seeded on 6-well plates. After $24 \mathrm{~h}$, when the cells were grown to $90-100 \%$ confluency, wounds were generated in the cells by scraping with a plastic tip across the cell monolayer. Phase-contrast images were recorded at the time of wounding $(0 \mathrm{~h})$ and at $24 \mathrm{~h}$ thereafter. Negative transfected cells served as controls. The mobility was calculated by the formula:

Migration ratio $=\left(\mathrm{Width}_{0 \mathrm{~h}}-\mathrm{Width}_{24 \mathrm{~h}}\right) / \mathrm{Width}_{0 \mathrm{~h}} \times 100 \%$ (formula 2).

Measurement of cell invasiveness. Cell invasiveness was examined using 6-well Transwell chambers and a reconstituted extracellular matrix membrane (Matrigel, USA). The cell invasion chambers were prepared by placing $100 \mu \mathrm{l}$ of a 1:8 dilution of Matrigel onto the filter, and incubating the filter at $37^{\circ} \mathrm{C}$ for $30 \mathrm{~min}$ to allow Matrigel polymerization. After radiotherapy, the cells were resuspended at $5 \times 10^{5}$ cells $/ \mathrm{ml}$ in serum-free medium. Afterwards, $200 \mu 1$ of cell suspension from each treatment group was added to the upper chambers. The chambers were incubated for $24 \mathrm{~h}$ in $37^{\circ} \mathrm{C}$ with $5 \% \mathrm{CO}_{2}$. The filters were then fixed in anhydrous ethanol and stained with crystal violet. The upper surfaces of the filters were scraped twice with cotton swabs to remove the non-migrated cells. The experiments were repeated in triplicate wells, and the migrated cells were counted microscopically (x200) in five different fields per filter.

Apoptosis assays. After radiotherapy, apoptosis was detected using the Annexin V-FITC Apoptosis Detection kit (KeyGene, Nanjing, China). The results are expressed as the percentage of apoptotic cells in all counted cells.

Xenograft model and treatment protocol. Female BALB/c nu/nu (athymic nude) mice (5-6 weeks of age) were obtained from Yangzhou University Animal Laboratory (China) and maintained on a 12-h light/12-h dark cycle, with food and water supplied ad libitum. U251 and RR-U251 cells (2x10 ${ }^{6}$ cells in $150 \mu \mathrm{l}$ PBS) were inoculated subcutaneously into the right flank of the mice. The tumors were allowed to grow to an average volume of $200 \mathrm{~mm}^{3}$ prior to initiation of therapy as described, then the tumor-bearing mice were randomly divided into 4 groups (U251, pcDNA-TPM1 U251, RR-U251 and pcDNA-TPM1 RR-U251; $n=6$ mice/group). Tumor size was determined by caliper measurement of two perpendicular diameters of the implant once every two days. Tumor volume was estimated by the formula $\mathrm{a} \times \mathrm{b}^{2} / 2$, where ' $\mathrm{a}$ ' is the long 
A

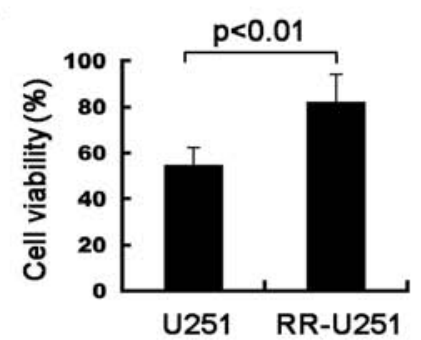

C

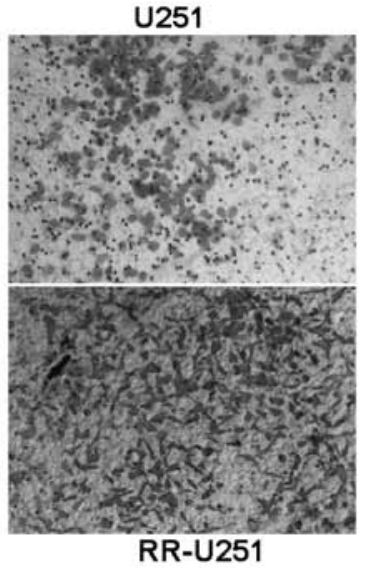

B

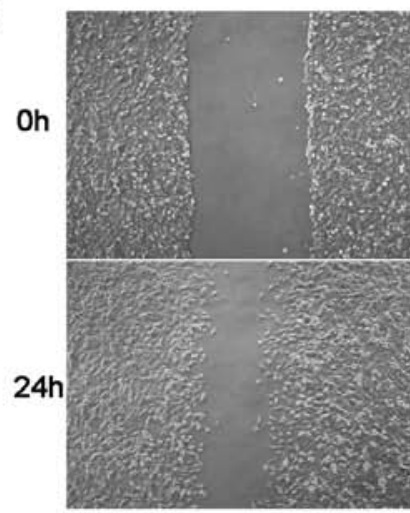

U251

D

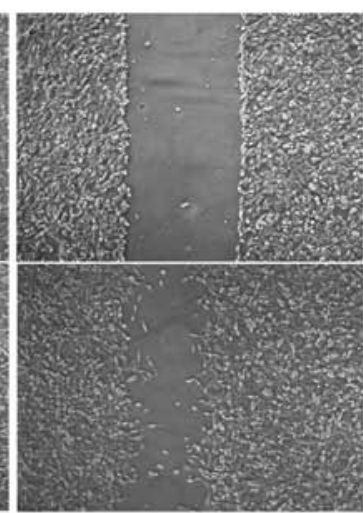

RR-U251
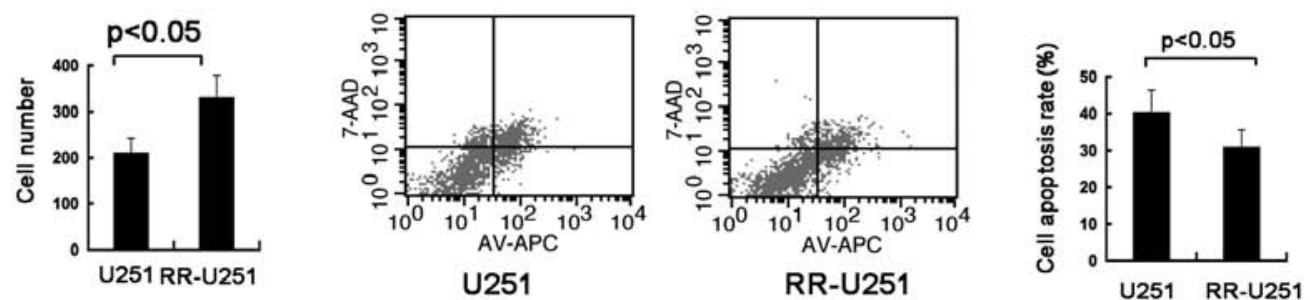

Figure 1. Establishment of the RR-U251 cells. After radiotherapy, (A) cell viability was evaluated in the U251 and RR-U251 cells. (B) Cell migration and (C) invasion abilities were assessed in the U251 and RR-U251 cells. (D) Cell apoptosis was examined in the U251 and RR-U251 cells. Data are representative of three independent experiments.

diameter and ' $b$ ' is the short diameter (in $\mathrm{mm}$ ). All protocols were approved by the Ethics Committee.

In vivo electrotransfection. Tumors were treated with an intratumoral injection of $20 \mu \mathrm{l}$ pcDNA-TPM1 and immediately thereafter electric pulses were applied $(8$ square wave pulses of amplitude $240 \mathrm{~V}$; amplitude over distance ratio $600 \mathrm{~V} / \mathrm{cm}$; duration $5 \mathrm{msec}$ at frequency $1 \mathrm{~Hz}$ ). Electric pulses were generated by electric pulse generator GT-01 (Faculty of Electrical Engineering, University of Ljubljana, Slovenia) and delivered through 2 parallel stainless steel electrodes with $4 \mathrm{~mm}$ distance between them. After $48 \mathrm{~h}$, all groups receive $5 \mathrm{~Gy}$ radiotherapy. After $48 \mathrm{~h}$, the next round of electrotransfection began. After 20 Gy radiotherapy, the mice were sacrificed, and the tumors were removed and submerged in $10 \%$ neutral buffered formalin for immunohistochemical analysis.

Immunohistochemistry. From each experimental group, 2 or 3 mice were sacrificed at day 2 post-treatment, and the tumors were excised. The tumors were fixed in IHC zinc fixative (BD Pharmingen and BD Biosciences) and embedded in paraffin. The immunohistochemical staining sections were incubated with rabbit polyclonal antibodies against murine TPM1 (ab55915; Abcam, Cambridge, MA, USA) at a dilution 1:300. A peroxidase-conjugated streptavidin-biotin system was used as the colorogenic reagent followed by hematoxylin counterstaining. The immunohistochemically stained slides were observed under light microscopy and from each slide 6 images of randomly selected areas of viable tumor tissue were captured with a camera.
Statistical analysis. Data were analyzed using the SPSS 17.0 software and are presented as mean \pm standard deviation (SD). Statistical analysis was carried out using one-sample Student's $\mathrm{t}$-test to compare differences between the treated cells and their appropriate controls. $\mathrm{P}<0.05$ was considered to indicate a statistically significant result.

\section{Results}

Establishment of the RR-U251 cells. RR-U251 cells were established from normal U251 cells, irradiated by a ${ }^{60} \mathrm{Co}$ source at $0.5 \mathrm{~Gy} / \mathrm{min}$ for $10 \mathrm{~min}$ each time until the accumulation was $60 \mathrm{~Gy}$. Then the radiosensitivity was characterized by measuring the cell viability and apoptosis, and migration and invasion ability after radiotherapy. The results showed that the cell viability was significantly increased in the irradiation-treated RR-U251 cells compared with the normal U251 cells (Fig. 1A). Similar results were also observed for the cell migration (Fig. 1B) and invasion assays (Fig. 1C). After radiotherapy, the cell apoptosis rate was higher in the U251 cells than that in the RR-U251 (Fig. 1D). All the results indicated that the radiosensitivity was decreased in the RR-U251 cells.

Modulating TPM1 expression by transfection. U251 and RR-U251 cells were transfected with the shRNA-TPM1 or pcDNA3.1-TPM1 duplexes to modulate the expression of TPM1. As shown in Fig. 2, the normal U251 and RR-U251 cells transfected with the shRNA-TPM1 duplexes showed a significantly lower expression of TPM1 as compared with the 
A

U251

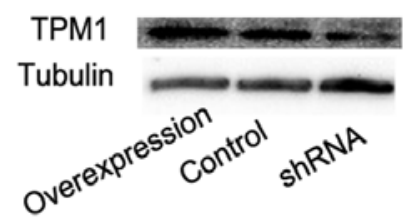

C

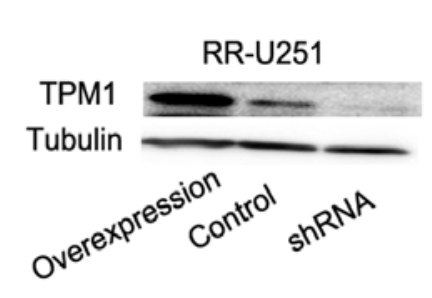

B

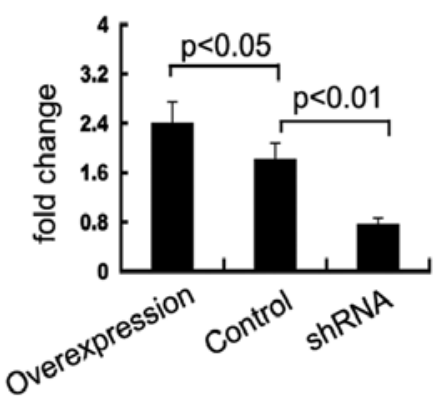

D

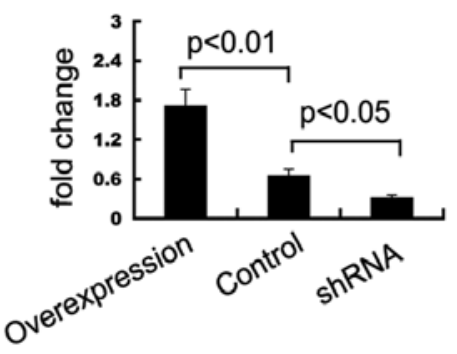

Figure 2. Modulation of the TPM1 expression in U251 and RR-U251 cells. Cells were transfected with GFP-shRNA or pcDNA3.1-TPM1. Western blotting was used to detect protein expression of TPM1 after transfection in the (A) U251 and (C) RR-U251 cells (C). Bar graphs show the level of TPM1 protein in the (B) U251 and (D) RR-U251 cells. $\alpha$-tubulin served as the loading control. Relative expression of TPM1 was determined by densitometry. Each experiment was performed three times.
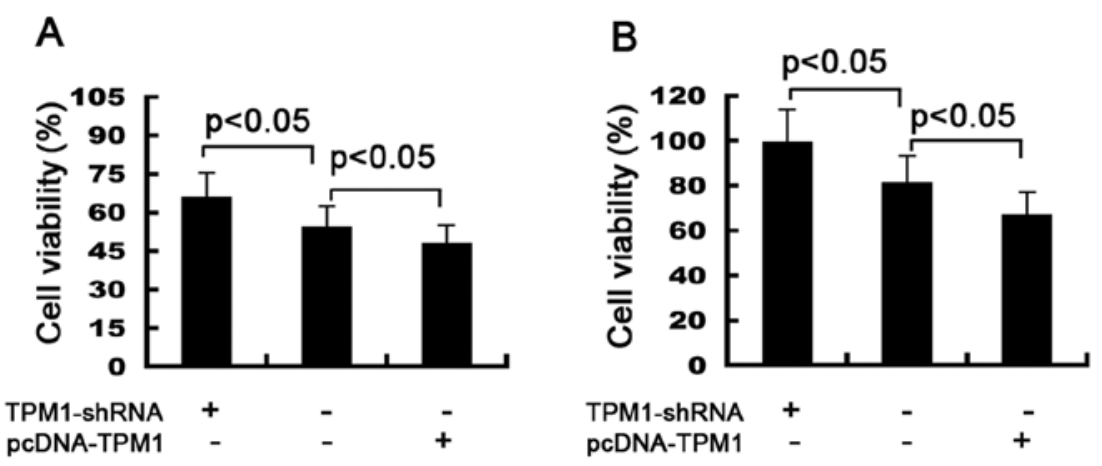

Figure 3. Effect of TPM1 on the proliferation of U251 and RR-U251 cells. (A) U251 and (B) RR-U251 cells transfected as indicated (control, TPM1 overexpressing group or TPM1-shRNA group) were treated by ${ }^{60} \mathrm{Co}$ once. After $48 \mathrm{~h}$, the proliferation ratio was determined by MTT assay. Data are representative of three independent experiments.
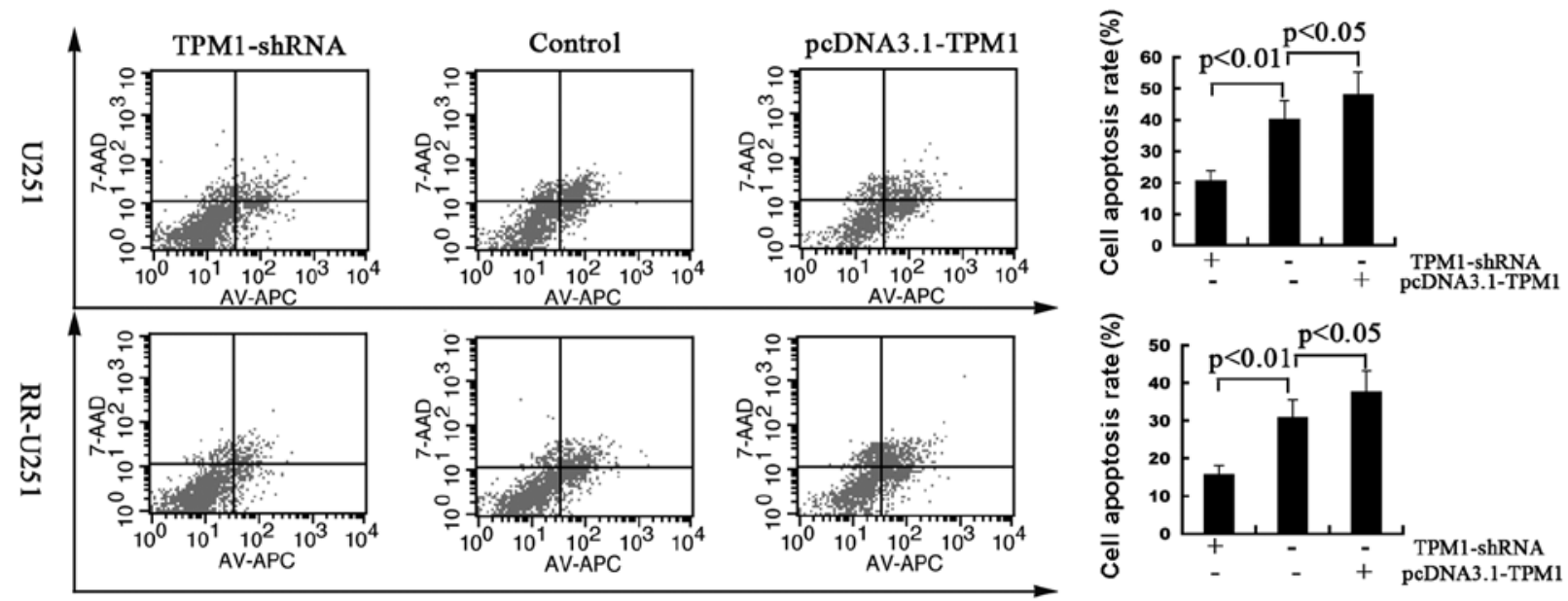

Figure 4. Analysis of cell apoptosis in the U251 and RR-U251 cells. U251 and RR-U251 cells transfected as indicated (control, TPM1 overexpressing group or TPM1-shRNA group) were treated by ${ }^{60} \mathrm{Co}$ once. Cells were then analyzed by flow cytometric analysis. 
A TPM1-shRNA

Control

pcDNA3.1-TPM1

oh
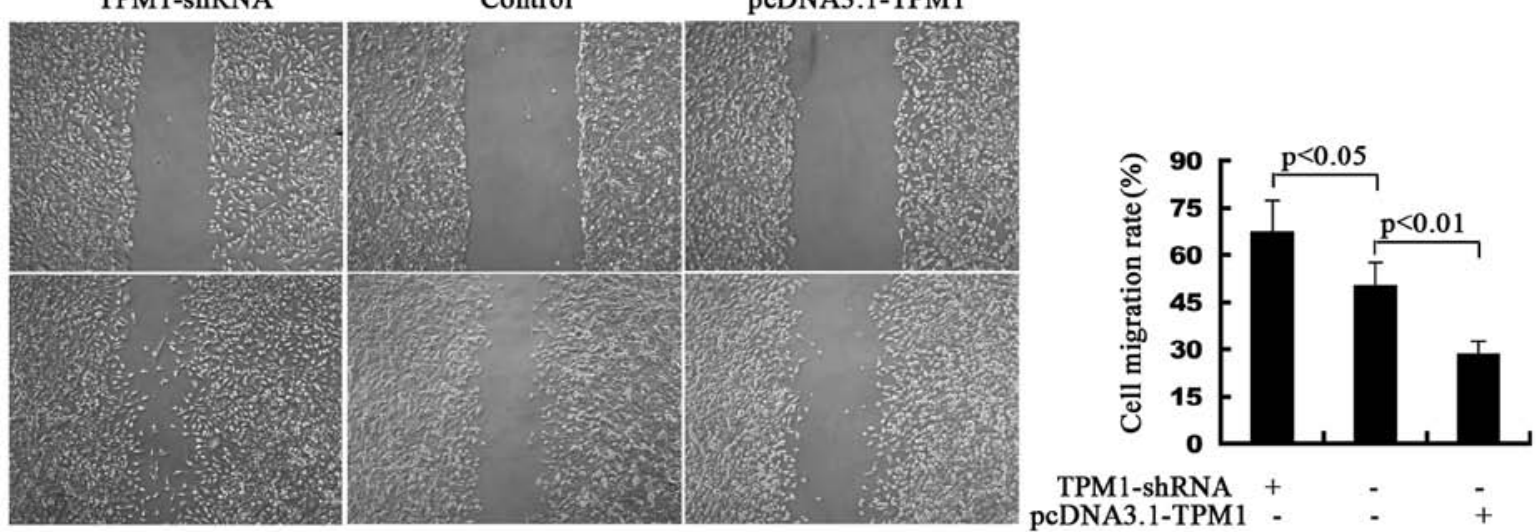

TPM1-shRNA + pcDNA3.1-TPM1 - $\quad-\quad+$

B
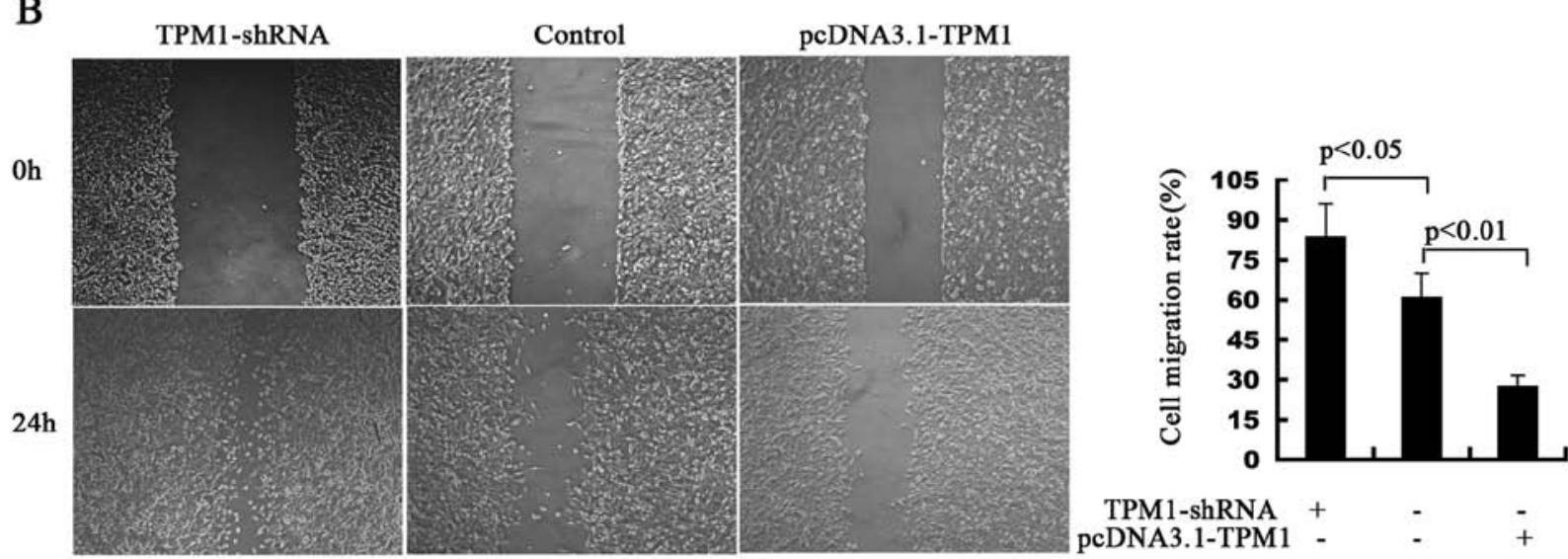

TPM1-shRNA + pcDNA3.1-TPM1 - $\quad-\quad+$

C
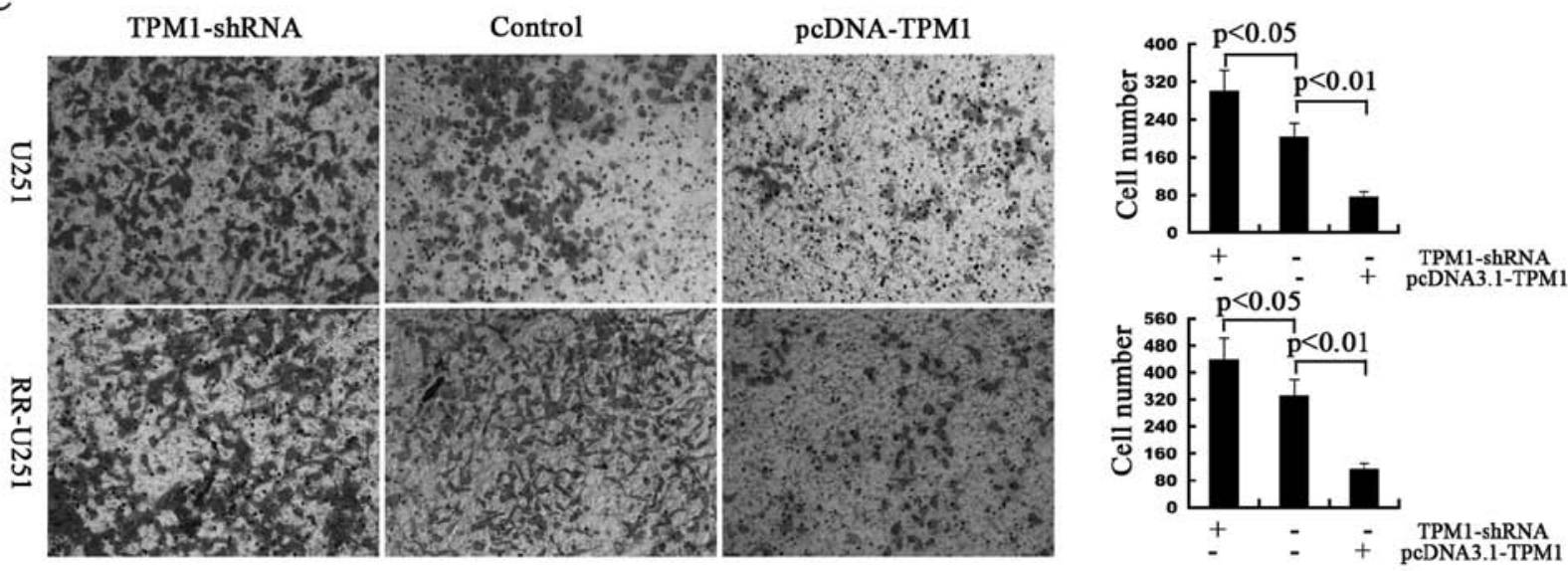

Figure 5. Effect of TPM1 on U251 cell migration and invasion ability. (A) U251 and (B) RR-U251 cells transfected as indicated (control, TPM1 overexpressing group or TPM1-shRNA group) were treated by ${ }^{60} \mathrm{Co}$ once. The monolayer cells were scratched using the tip of a $10 \mu 1$ pipette to create the wound line. Images were captured at 0 and $24 \mathrm{~h}$ at the same site using a fluorescence microscope. (C) U251 and RR-U251 cells transfected as indicated (control, TPM1 overexpressing group or TPM1-shRNA group) were treated by ${ }^{60} \mathrm{Co}$ once. The cell invasion ability was evaluated by the Transwell culture chamber system. Each experiment was performed three times.

expression in the cells transfected with an irrelevant sequence control $(\mathrm{P}<0.05)$. The cells transfected with the high expression plasmid, pcDNA3.1-TPM1, showed a higher TPM1 expression as compared with the expression in the controls as confirmed by western blotting $(\mathrm{P}<0.05)$.

Radiotherapy and cell viability. In the present study, the cells were exposed to 5-Gy of $\gamma$-rays to investigate the effects on cell proliferation. There are no previous studies on the relation- ship between TPM1 and radiotherapy in glioma. The results demonstrated that RR-U251 and U251 cells transfected with TPM1-shRNA exhibited a significant increase in cell viability compared with the controls $(\mathrm{P}<0.05$, Fig. 3$)$. On the contrary, upregulation of TPM1 decreased cellular proliferation in the U251 cells when compared with that in the RR-U251 cells $(\mathrm{P}<0.05)$. These results demonstrated that TPM1-shRNA promoted cellular proliferation in the glioblastoma cells and decreased the radiosensitivity. 

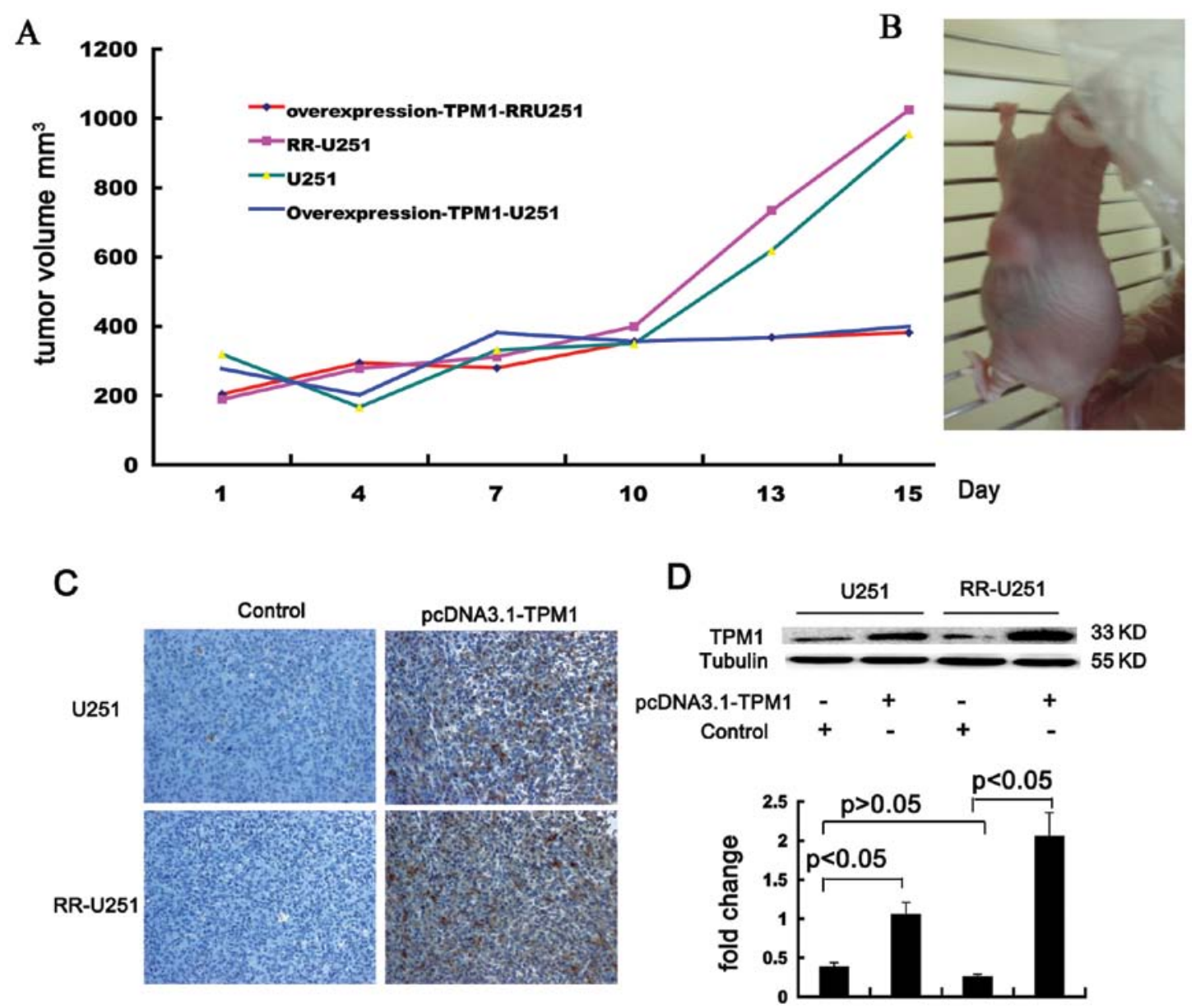

Figure 6. Effect of pcDNA3.1-TPM1 transfection together with irradiation on the growth of U251 cell xenografts in nude mice. Six mice were used for each group. Every two days, TPM1-shRNA was electroporated and mice were administered a radiation dose of $20 \mathrm{~Gy}$. The two perpendicular diameters were measured every two days and tumor volume was estimated. (A) The curve of tumor growth was plotted according to tumor volume. (B) Tumor in a nude. (C) Immunohistochemistry and (D) western blotting were used to detect to protein expression of TPM1 in the different tumors transfected with TPM1-shRNA. $\alpha$-tubulin served as the loading control. Relative expression of TPM1 was determined by densitometry. Each experiment was performed three times.

Radiotherapy and TPM1 induce apoptosis in the glioma cells. To confirm that the expression of TPM1 is associated with apoptosis, we examined the apoptosis of the cells by flow cytometry. The results obviously demonstrated that after 5 Gy radiation there was a significant decrease in apoptosis in the RR-U251 and U251 cells transfected with TPM1-shRNA compared with the cells transfected with the control $(\mathrm{P}<0.05)$. In contrast, upregulation of TPM1 increased the apoptosis of the cancer cells, while U251 cells showed an increase in apoptosis compared with the RR-U251 cell group ( $\mathrm{P}<0.05$, Fig. 4). These data confirm that TPM1 plays an apoptotic role in glioblastoma cells.

Radiotherapy and cell migration and cell invasion ability. To examine whether modulation of TPM1 affects the migration and invasion capacity of glioblastoma cells, wound healing and Transwell assays were conducted. The results clearly revealed that the absence of TPM1 also increased the migration of the U251 cells compared with the RR-U251 cells $(\mathrm{P}<0.05$, Fig. 5A and B). We also found identical effects of TPM1-shRNA exerted on the RR-U251 cell line compared with the control group ( $\mathrm{P}<0.05$, Fig. 5B). In the rescue experiment, overexpression of TPM1 obviously inhibited the invasion and migration of the glioma cells. In parallel, U251 cells transfected with
TPM1-shRNA exhibited enhanced invasive ability compared with the control group ( $\mathrm{P}<0.05$, Fig. $5 \mathrm{C})$. In addition, the invasive ability of the RR-U251 cells was increased when compared with the U251 cells. These results above strongly suggest that TPM1 is an important factor involved in the migration and invasion of glioblastoma cells .

Radiotherapy and TPM1 in the tumor xenografts. To examine the effect of TPM1 on radiosensitivity, the nude mouse tumor volumes were determined. As shown in Fig. 6, overexpression of TPM1 reduced the tumor volume. The immunohistochemistry and western blotting results also verified that after pcDNA3.1-TPM1 treatment the level of TPM1 was higher than that in the control groups. However, there was no significant difference between the U251 and RR-U251 groups in the in vivo experiment, which requires further in-depth study.

\section{Discussion}

Human gliomas are one of the most aggressive and malignant types of cancers, and present a great challenge for medical personnel due to the poor prognosis and frequent recurrence. At present, the main therapeutic method for glioma is radiotherapy. However, the efficacy of this therapeutic modality 
is often limited by the occurrence of radioresistance. Thus, extensive study has been conducted in the area of radioresistance (17).

Multiple factors limit the efficacy of radiation. These include changes in the cell cycle distribution, activation of oncogenes, and inactivation of tumor-suppressor genes (18-20). Another potential reason for the inefficacy of radioresistance may be the occurrence of glioma stem cells $(21,22)$. TPM1 functions as a tumor-suppressor gene. Several studies have reported that high-grade tumors of the breast, prostate, bladder and brain express significantly lower levels of HMW TMs compared to normal tissues $(10,23)$. In our previous study, we found that in RR-U251 cells, the expression of TPM1 was low suggesting that deregulation of TPM1 can lower the efficacy of radiotherapy.

Radioresistance can be a consequence of a deregulated cell cycle, altered rate of apoptosis, or altered frequency of DNA repair (24-26). A previous study revealed that evasion of apoptosis is one of the hallmarks of glioblastoma (27) and also contributes to chemoresistance or radioresistance since therapy-induced cytotoxicity is mediated, to a large extent, by induction of cell death, including apoptosis in cancer cells (28). Our data found that transfection with shTPM1 in the cancer cells inhibited apoptosis and mediated radioresistance. However, radiation is also able to stimulate other important signaling pathways which regulate cell survival, proliferation and apoptosis $(29,30)$. To date, the specific signaling pathway involved in the effect on radiotherapy for unresectable glioma remains unknown.

The actin cytoskeleton plays an important role in the regulation of cell proliferation, apoptosis, cell migration, invasion and anchorage-independent growth. Actin filament dynamics and reorganization are spatiotemporally regulated by numerous actin-binding proteins and upstream signaling molecules, which cooperatively control the assembly and disassembly of actin filaments, and construction and destruction of actin filament-based supramolecular cytoskeletal structures, such as filopodia, lamellipodia, invadopodia, stress fibers and cortical actin networks (31-33). Actin filament dynamics and reorganization are necessary for the formation and progression of tumors, as well as recurrence and metastasis. TMs are actin-stabilizing proteins consisting of two $\alpha$-helical chains arranged as a coiled-coil that bind along actin filaments and prevent the binding of actin-destabilizing proteins such as cofilin and gelsolin $(34,35)$. TMs influence cell migration and invasion $(36,37)$, and extensive invasion is one of the key features of glioma, and is often associated with a high incidence of post-resection recurrence and poor prognosis (38). Our data also suggested that silencing of TPM1 induces cancer cell migration and invasion, which influence the efficacy of radiotherapy.

Importantly, in the in vivo experiment, overexpression of TPM1 inhibited tumor growth. Yet, there was no significant difference between the U251 and RR-U251 cells. This indicates a new study dimension.

In summary, silencing of the TPM1 gene induced proliferation, migration, invasion and apoptosis of human glioblastoma, which indirectly influenced the radiosensitivity. We firstly revealed the crucial role of TPM1 in the radiotherapy of human glioblastoma cells. These conclusions were consistent with our previous assumptions. Unfortunately, many important questions remain unanswered. The mechanism underlying the TPM1 and radioresistance requires further investigation. In addition, the reason for the discrepant results in the in vitro and in vivo study requires further research.

\section{Acknowledgements}

This study was supported by grant NSFC81172390 from the National Science Foundation of China and by grant ZKX10021 from the Health Bureau of Nanjing, China.

\section{References}

1. Louis DN, Ohgaki H, Wiestler OD, Cavenee WK, Burger PC, Jouvet A, Scheithauer BW and Kleihues P: The 2007 WHO classification of tumours of the central nervous system. Acta Neuropathol 114: 97-109, 2007.

2. Louis DN: Molecular pathology of malignant gliomas. Annu Rev Pathol 1: 97-117, 2006.

3. Stupp R, Hegi ME, Mason WP, van den Bent MJ, Taphoorn MJ, Janzer RC, Ludwin SK, Allgeier A, Fisher B, Belanger K, et al; European Organisation for Research and Treatment of Cancer Brain Tumour and Radiation Oncology Groups; National Cancer Institute of Canada Clinical Trials Group: Effects of radiotherapy with concomitant and adjuvant temozolomide versus radiotherapy alone on survival in glioblastoma in a randomised phase III study: 5-year analysis of the EORTC-NCIC trial. Lancet Oncol 10: 459-466, 2009.

4. Glioma Meta-Analysis Trialists (GMT) Group: Chemotherapy for high-grade glioma. Cochrane Database Syst Rev 4: CD003913, 2002 .

5. Ohuchida K, Mizumoto K, Murakami M, Qian LW, Sato N, Nagai E, Matsumoto K, Nakamura T and Tanaka M: Radiation to stromal fibroblasts increases invasiveness of pancreatic cancer cells through tumor-stromal interactions. Cancer Res 64: 3215-3222, 2004.

6. Kwiatkowska A and Symons M: Signaling determinants of glioma cell invasion. Adv Exp Med Biol 986: 121-141, 2013.

7. Stupp R, Mason WP, van den Bent MJ, Weller M, Fisher B, Taphoorn MJ, Belanger K, Brandes AA, Marosi C, Bogdahn U, et al; European Organisation for Research and Treatment of Cancer Brain Tumor and Radiotherapy Groups; National Cancer Institute of Canada Clinical Trials Group: Radiotherapy plus concomitant and adjuvant temozolomide for glioblastoma. $\mathrm{N}$ Engl J Med 352: 987-996, 2005.

8. Frosina G: DNA repair and resistance of gliomas to chemotherapy and radiotherapy. Mol Cancer Res 7: 989-999, 2009.

9. Zhu Z, Kleeff J, Kayed H, Wang L, Korc M, Büchler MW and Friess $\mathrm{H}$ : Nerve growth factor and enhancement of proliferation, invasion, and tumorigenicity of pancreatic cancer cells. Mol Carcinog 35: 138-147, 2002.

10. Raval GN, Bharadwaj S, Levine EA, Willingham MC, Geary RL, Kute T and Prasad GL: Loss of expression of tropomyosin-1, a novel class II tumor suppressor that induces anoikis, in primary breast tumors. Oncogene 22: 6194-6203, 2003.

11. Pawlak G, McGarvey TW, Nguyen TB, Tomaszewski JE, Puthiyaveettil R, Malkowicz SB and Helfman DM: Alterations in tropomyosin isoform expression in human transitional cell carcinoma of the urinary bladder. Int J Cancer 110: 368-373, 2004.

12. Yager ML, Hughes JA, Lovicu FJ, Gunning PW, Weinberger RP and O'Neill GM: Functional analysis of the actin-binding protein, tropomyosin 1, in neuroblastoma. Br J Cancer 89: 860-863, 2003.

13. Choi C, Kim D, Kim S, Jeong S, Song E and Helfman DM: From skeletal muscle to cancer: insights learned elucidating the function of tropomyosin. J Struct Biol 177: 63-69, 2012.

14. Mahadev K, Raval G, Bharadwaj S, Willingham MC, Lange EM, Vonderhaar B, Salomon D and Prasad GL: Suppression of the transformed phenotype of breast cancer by tropomyosin-1. Exp Cell Res 279: 40-51, 2002.

15. Jemal A, Bray F, Center MM, Ferlay J, Ward E and Forman D: Global cancer statistics. CA Cancer J Clin 61: 69-90, 2011. 
16. Aiyar SE, Park H, Aldo PB, Mor G, Gildea JJ, Miller AL, Thompson EB, Castle JD, Kim S and Santen RJ: TMS, a chemically modified herbal derivative of resveratrol, induces cell death by targeting Bax. Breast Cancer Res Treat 124: 265-277, 2010.

17. Ahmed R, Oborski MJ,Hwang M,Lieberman FS and Mountz JM: Malignant gliomas: current perspectives in diagnosis, treatment, and early response assessment using advanced quantitative imaging methods. Cancer Manag Res 6: 149-170, 2014.

18. Maity A, McKenna WG and Muschel RJ: The molecular basis for cell cycle delays following ionizing radiation: a review. Radiother Oncol 31: 1-13, 1994.

19. Pawlik TM and Keyomarsi K: Role of cell cycle in mediating sensitivity to radiotherapy. Int J Radiat Oncol Biol Phys 59: 928-942, 2004.

20. Hendry JH: Radiation biology and radiation protection. Ann ICRP 41: 64-71, 2012.

21. Piccirillo SG and Vescovi AL: Brain tumour stem cells: possibilities of new therapeutic strategies. Expert Opin Biol Ther 7: 1129-1135, 2007

22. Xie Z and Chin LS: Molecular and cell biology of brain tumor stem cells: lessons from neural progenitor/stem cells. Neurosurg Focus 24: E25, 2008.

23. Pawlak JK: Brain teaser: facial swelling and tenderness. Aust Fam Physician 33: 634, 2004.

24. Sharma S, Hicks JK, Chute CL, Brennan JR, Ahn JY, Glover TW and Canman CE: REV1 and polymerase $\zeta$ facilitate homologous recombination repair. Nucleic Acids Res 40: 682-691, 2012.

25. Xie C, Wang H, Cheng H, Li J, Wang Z and Yue W: RAD18 mediates resistance to ionizing radiation in human glioma cells. Biochem Biophys Res Commun 445: 263-268, 2014.

26. McLaughlin N, Annabi B, Bouzeghrane M, Temme A, Bahary JP, Moumdjian R and Béliveau R: The survivin-mediated radioresistant phenotype of glioblastomas is regulated by RhoA and inhibited by the green tea polyphenol (-)-epigallocatechin-3-gallate. Brain Res 1071: 1-9, 2006.
27. Kögel D, Fulda S and Mittelbronn M: Therapeutic exploitation of apoptosis and autophagy for glioblastoma. Anticancer Agents Med Chem 10: 438-449, 2010.

28. Fulda S and Debatin KM: Extrinsic versus intrinsic apoptosis pathways in anticancer chemotherapy. Oncogene 25: 4798-4811, 2006.

29. Tessner TG, Muhale F, Riehl TE, Anant S and Stenson WF: Prostaglandin E2 reduces radiation-induced epithelial apoptosis through a mechanism involving AKT activation and bax translocation. J Clin Invest 114: 1676-1685, 2004.

30. Toulany M, Dittmann K, Fehrenbacher B, Schaller M, Baumann $M$ and Rodemann HP: PI3K-Akt signaling regulates basal, but MAP-kinase signaling regulates radiation-induced XRCC1 expression in human tumor cells in vitro. DNA Repair (Amst) 7: 1746-1756, 2008

31. Cooper JA and Schafer DA: Control of actin assembly and disassembly at filament ends. Curr Opin Cell Biol 12: 97-103, 2000.

32. Pollard TD and Borisy GG: Cellular motility driven by assembly and disassembly of actin filaments. Cell 112: 453-465, 2003.

33. Jaffe AB and Hall A: Rho GTPases: Biochemistry and biology. Annu Rev Cell Dev Biol 21: 247-269, 2005.

34. Perry SV: Vertebrate tropomyosin: Distribution, properties and function. J Muscle Res Cell Motil 22: 5-49, 2001

35. Ono S and Ono K: Tropomyosin inhibits ADF-cofilin-dependent actin filament dynamics. J Cell Biol 156: 1065-1076, 2002.

36. Hitchcock-DeGregori SE, Greenfield NJ and Singh A: Tropomyosin: Regulator of actin filaments. Adv Exp Med Biol 592: 87-97, 2007.

37. Bugyi B and Carlier MF: Control of actin filament treadmilling in cell motility. Annu Rev Biophys 39: 449-470, 2010

38. Caraglia $\mathrm{M}$ and Addeo R: Editorial: target therapy in brain tumours and metastases. Curr Cancer Drug Targets 12: 185, 2012. 